\title{
Lutte intégrée contre les parasitoses animales tropicales *
}

\author{
G. Uilenberg 1
}

\section{Mots clés}

Parasitose animale - Lutte intégrée Résistance aux produits chimiques Coût - Environnement - Rentabilité Pays en développement.
La lutte contre les parasitoses tropicales des animaux de rente a été basée pendant longtemps principalement sur l'emploi de médicaments et de pesticides. Cela reste vrai pour les ectoparasites dont le cycle se déroule entièrement sur l'hôte, par exemple les acariens des gales et les poux. La lutte contre ce type de parasite est habituellement simple. On peut en général les éliminer du troupeau par une ou deux applications d'un composé spécifique et prévenir les réinfestations par des mesures sanitaires et par une surveillance. Un nouveau traitement ne s'impose qu'en cas de réinfestation. D'autres exemples dẹ parasites qui ne quittent pas

* Cet article est publié conjointement en français dans ce numéro de la Revue d'élevage et de médecine vétérinaire des pays tropicaux et en anglais dans le vol. $28 \mathrm{n}^{\circ} 4$ de Tropical animal health and production. Il est issu d $\mathrm{d}^{2}$ une conférence ayant eu lieu le 7 décembre 1995 à l'occasion du congrès d'hiver 1995-1996 de la Société française de parasitologie à l'Ecole nationale vétérinaire d'Alfort, France (16).

1. " $\Lambda$ Surgente", Roùte du Port, 20130 Cargèse, Corse leur hôte sont l'agent de la trichomonose bovine, Trichomonas foetus, et celui de la dourine, Trypanosoma equiperdum; après avoir obtenu la guérison de l'animal par un traitement médicamenteux, ou après élimination des animaux atteints du troupeau, il suffit d'empêcher des réinfections par d'autres mesures.

Mais la majorité des parasites ont des stades en dehors de leurs hôtes, soit dans l'environnement (comme beaucoup d'helminthes, de coccidies et d'autres protozoaires intestinaux, et la grande majorité des arthropodes piqueurs), soit chez des vecteurs ou des hôtes intermédiaires (tels que tous les parasites transmis par des arthropodes, heaucoup de coccidies sensu lato, et beaucoup d'helminthes). La guérison de l'hôte ne peut empêcher des réinfestations ou des réinfections à partir de l'environnement, des vecteurs ou des hôtes intermédiaires, et la lutte a traditionnellement été basée sur des traitements réguliers, systématiques, sans fin et sans espoir d'éradication. Les raisons suivantes sont à l'origine d'un changement d'approche, qui se dessine ces dernières années pour différentes raisons : 


\section{1) Résistance}

Il pourrait bien y avoir des mutants naturels dans les populations de parasites contre tous les antiparasitaires actuels et futurs. Après tout, les arthropodes ct les nématodes parasites ont dû fairc face depuis des millions d'années à des pesticides naturels, surtout ceux produits par des plantes, et ont dû développer une stratégie basée sur des mutations afin de survivre.

Quoi qu'il en soit, la plupart des parasites importants des animaux ont actuellement développé une résistance à une ou à plusieurs familles de molécules, et parfois même à tous les composés disponibles dans le commerce, tandis que le développement de nouveaux médicaments et pesticides est presque au point mort, en particulier pour les parasitoses tropicales ; plusieurs molécules ont même été retirées du marché.

Pour ne mentionner que quelques exemples :

Une résistance des trypanosomes africains des animaux domestiques a été observée contre les quelques composés actuellement disponibles et constitue un problème de première importance.

Le problème des résistances contre les insecticides chez beaucoup d'insectes piqueurs est bien connu. Les glossines, vecteurs des trypanosomoses africaines, ne sont heureusement pas (encore) concernées, sans doute parce qu'il existe peu de chances de rencontrer les mutants nécessaires à cause du faible nombre de descendants.

La résistance des tiques aux acaricides est un problème majeur. Là où le détiquage est intensif, des résistances aux acaricides utilisés et aux molécules apparentées se sont développées. Certaines souches de la tique Boophilus microplus en Australie sont à présent résistantes à tous les acaricides disponibles dans le commerce, et de ce fait les Australiens ont été des pionniers de méthodes alternatives pour la lutte contre les tiques.

Les helminthes d'animaux ont également développé des résistances à plusieurs anthelminthiques, y compris aux endectocides tels que l'ivermectine, la moxidectine, le closantel...

Par ailleurs, la résistance aux anticoccidiens constitue une difficulté énorme pour l'élevage avicole.

Un exemple frappant en médecine humaine est bien sûr celui du paludisme où la lutte ne peut plus être basée uniquement sur l'utilisation d'insecticides et sur la chimioprophylaxie, à cause des multirésistances aussi bien chez les vecteurs que chez les Plasmodium.

\section{2) Le coût financier}

L'industrie pharmaceutique souscrit évidemment au principe de coût/bénéfice, sans quoi elle ne survivrait pas. Or, le coût de la recherche est en augmentation constante. La réglementation sur la toxicité chronique et sur les résidus des composés est de plus en plus stricte et leur admission officielle devient de plus en plus difficile et coûteuse. En conséquence logique, chaque nouvelle molécule est plus chère que les précédentes. Mais le marché des pays en voie de développement est limité financièrement et en volume, et n'incite pas l'industrie à investir les sommes énormes requises pour développer de nouveaux produits contre les parasitoses tropicales.

Dans beaucoup de pays en voie de développement, le problème de manque de devises est un facteur important. La plupart des médicaments et des pesticides sont fabriqués dans les pays industrialisés et la part des devises que les gouvernements, peuvent ou veulent utiliser pour leur importation est souvent très limitée. (Les pays de la zone franc constituent une exception étant donné que le taux d'échange du franc CFA avec le franc français est fixe et garanti par la France. Bien entendu, cela n'enlève pas les limitations financières quantitatives, surtout après la dévaluation récente du franc CFA.)

Depuis la mise sur le marché de l'isométamidium, il y a une trentaine d'années, un seul autre composé contre les trypanosomoses animales africaines est apparu dans le commerce, le Mel Cy (Cymelarsan $\left.{ }^{\circledR}\right)$, contre Trypanosoma evansi, et le nombre de trypanocides est extrêmement limité. La situation n'est pas meilleure en ce qui concerne la maladie du sommeil humaine, car le nombre de médicaments autorisés est encore plus limité ; bien qu'un nouveau traitement de cette maladie existe (DL- $\alpha$-difluorométhylornithine $=$ DFMO = eflornithine), ce traitement est trop long et son prix trop élevé dans les conditions africaines. Le nombre de cas de trypanosomose humaine africaine est par ailleurs beaucoup trop faible pour justifier de programmes spéciaux de recherche par l'industrie pharmaceutique pour le développement de trypanocides nouveaux. Même le marché de la trypanosomose animale est trop limité pour que l'industrie y consacre un effort réel. Les pays et les organisations de donateurs ont un rôle évident dans ce domaine.

La mise au point du parvaquone et de l'halofuginone pour le traitement des theilérioses virulentes (Theileria parva et $T$. annulata) vers la fin des années 1970 a créé un grand espoir, car aucun médicament efficace n'était disponible auparavant. Malheureusement, le prix de vente du parvaquone et de son successeur, le buparvaquone, est beaucoup trop élevé pour le marché africain ; malgré ce prix élevé, il semble bien que la compagnie concernéc ne pourra jamais récupérer son investissement, car lc marché est trop limité. Le lactate d'halofuginone n'a pas connu de succès commercial à cause de problèmes associés à sa fabrication, son administration et sa toxicité ; son prix n'aurait peut-être pas été excessif, car l'halofuginone était déjà en vente contre les coccidioses.

Le marché des médicaments contre les babésioses animales est limité, aussi bien dans les pays industrialisés que dans ceux en voie de développement. Quelques composés ont même été retirés du marché par les fabricants, soit complètement, soit dans certains pays, pour des raisons économiques.

L'industrie pharmaceutique est obligée d'investir des sommes énormes si elle veut développer de nouvelles familles d'acaricides contre les tiques. Mais la récupération de tels investissements devient de plus en plus problématique, car ce marché est aussi secondaire, même dans les pays relativement riches, comparé à celui des pesticides utilisés contre les parasites de cultures. Il semble bien que nous soyons à peu près condamnés à vivre avec ce qui existe actuellement. Aussi les experts s'interrogent-ils sur la meilleure stratégie pour retarder et gérer les résistances.

Etant donné que le développement de résistances contre les produits antiparasitaires semble inéluctable, l'industrie hésite à investir dans la recherche de nouvelles molécules. La durée pendant laquelle une nouvelle molécule sera efficace est imprévisible, mais certainement limitée. Le marché pour les anthelminthiques est certes relativement lucratif, car il est mondial et concerne toutes les espèces d'animaux domestiques ; néanmoins, des prix plus élevés affectent non seulement les éleveurs des pays en voie de développement, mais aussi ceux des pays industrialisés, pour lesquels la marge de bénéfice est souvent très réduite et chaque centime compte. Les composés antiparasitaires à large spectre sont bien sûr les plus attrayants, comme les endectocides récents, actifs contre les arthropodes et les nématodes, mais des résistances sont déjà connues chez quelques espèces de parasites. 


\section{3) Problèmes environnementaux et toxicologiques}

Un certain degré de pollution liée à l'utilisation sur les animaux d'insecticides et d'acaricides est inévitable, surtout lorsque l'application est régulière et fréquente. Le problème le plus important se pose lors du renouvellement du liquide dans les bains ou dans les réservoirs des couloirs d'aspersion ; que faire du liquide périmé ? La plupart des produits sont néfastes pour la vie aquatique et la pollution persistante qu'engendrent l'arsenic et les organochlorés autour d'anciens bains est d'actualité notoire aux Etats-Unis et en Australie par exemple.

Les insecticides pulvérisés sur la végétation contre les glossines peuvent avoir des effets négatifs sur l'environnement, mais ces effets sont habituellement passagers, car l'application n'est pas ou peu répétée. L'utilisation d'insecticides sur les cultures est beaucoup plus lourde et donc nuisible.

Les résidus de médicaments et de pesticides dans les produits animaux (viande, lait, laine...) constituent un problème universel, d'une très grande importance pour la santé publique.

Même les anthelminthiques peuvent être à l'origine de pollution. Notamment, on sait que l'ivermectine affecte la faune des arthropodes dans la bouse de vaches, et peut tuer les bousiers, ce qui retarde le recyclage des excréments. Le problème est réel en Australie où les bousiers spécifiques des fèces de ruminants faisaient défaut à l'origine et sont importés.

\section{4) La vulnérabilité des populations animales sous traitement antiparasitaire intensif et régulier}

A titre d'exemple, les bovins traités régulièrement et efficacement contre les tiques restent sensibles à toutes les maladies transmises par les tiques locales. Si les traitements sont interrompus pour une raison ou une autre (résistance à l'acaricide utilisé, rupture de l'approvisionnement d'acaricide, panne des installations, ou bien émeutes ou guerres civiles), des pertes très importantes peuvent se produire (12).

\section{LA LUTTE INTEGREE}

Elle consiste à utiliser de façon optimale toutes les méthodes de lutte disponibles de façon flexible, adaptées aux circonstances locales et aux réalités économiques. Elle peut comprendre des méthodes biologiqucs, immunologiques, mécaniques, génétiques, mais également chimiques, tandis que les procédures sanitaires et la surveillance restent valables. L'approche n'est pas vraiment nouvelle, il existe de nombreux exemples de longue date de lutte antiparasitaire par des mesures autres que l'utilisation de médicaments et de pesticides. Mais à cause des raisons mentionnées, l'accent est actuellement moins sur ces composés et plus sur une approche multidisciplinaire (voir par excmple Young ct coll., (17)). Néanmoins, la lutte rationnelle intégrée reste souvent encore un but plus ou moins lointain à atteindre; les méthodes potentielles ne sont pas toujours à la portée des techniciens et encore moins des populations rurales, et certaines méthodes n'ont pas encore fait preuve de leur efficacité et de leur rentabilité dans les conditions de terrain.

Ce qui suit est une revue de quelques composants actuels ou potentiels de la lutte intégrée pour certains groupes de parasites. II n'est pas dans notre intention d'être exhaustif ; les quelques références bibliographiques ne sont données qu'à titre d'exemples. Un numéro spécial de la Revue scientifique et technique de l'office in- ternational des épizooties traite en grande partie de ce domaine en ce qui concerne les ectoparasites (14).

\section{Lutte immunologique}

Il y a toujours bien peu d'exemples de vaccins appliqués contre des parasites. En ce qui concerne les helminthes, il existe un vaccin commercial contre la bronchite vermineuse (Dictyocaulus viviparus) des bovins, par administration de larves irradiées. Le commerce propose également des vaccins atténués contre des coccidioses aviaires.

La plupart des procédures d'immunisation utilisées dans les pays tropicaux, peu nombreuses d'ailleurs, ne sauraient guère intéresser l'industrie privée, car le marché est trop limité et le plus souvent pauvre, les procédures sont souvent compliquées, difficilement standardisables et comportent trop de risques pour intéresser le commerce. S'agissant de maladies à hématozoaires, comme les babésioses et les theilérioses, l'inoculation de l'organisme causal vivant est suivie d'un traitement spécifique s'il n'est pas atténué. Les risques de contamination, surtout virale, sont récls, ct l'utilisation d'organismes virulents comporte d'autres risques évidents. L'utilisation de ces méthodes est principalement limitée aux laboratoires gouvernementaux.

Plusieurs équipes étudient la possibilité d'utiliser des méthodes plus modernes d'immunisation contre les parasites mais jusqu'à présent les résultats pratiques sont bien minces. Il existe en France un vaccin commercial contre une babésiose (canine), basé sur des antigènes solubles de culture. Deux vaccins recombinants sont commercialisés depuis peu contre la tique Boophilus microplus, mais leur efficacité et leur rentabilité restent à démontrer par une utilisation à plus vaste échelle.

Un parasite est un organisme bien plus complexe qu'une bactérie ou un virus, même si les virus posent parfois des problèmes énormes dans ce domaine, comme notamment les virus de la grippe et du SIDA.

Néanmoins, l'avancée des biotechnologies est rapide, et on peut raisonnablement s'attendre à des progrès spectaculaires dans un avenir plus ou moins proche.

Dans le cas des méthodes immunologiques, il faut certainement parler aussi d'un terrain qui dépasse celui de l'immunisation artificielle. Certains individus chez les animaux, comme chez l'homme d'ailleurs, développent une meilleure réponse immunitaire à une infestation parasitaire que d'autres individus de la même population. De même, certaines populations et races tolèrent en moyenne un parasitisme donné mieux que d'autres. Les taurins et les petits ruminants trypanotolérants d'Afrique de l'Ouest sont connus depuis longtemps, et une certaine trypanotolérance a plus récemment été mise en évidence chez certaines populations de zébus de l'Afrique de l'Est (11). Les populations de bovins qui ont vécu depuis longtemps dans des régions endémiques de babésioses, theilérioses, etc. ne souffrent guère de ces infections, à l'opposé des bovins importés. Non seulement elles supportent bien mieux ces infections, mais également l'infestation par les tiques. Une tolérance relative vis-à-vis des infestations par des helminthes existe aussi par exemple chez certaines races de petits ruminants. Il s'agit normalement d'une sélection naturelle dans les régions endémiques, mais une observation récente indique qu'un gc̀nc responsable d'une résistance aux tiques pourrait également se retrouver fortuitement chez une population bovine originaire d'ailleurs (6).

Malheureusement, la productivité de la plupart de ces races "tolérantes" laisse à désirer et se situc loin en-dessous de celle du bétail 
sensible importé. On est véritablement pris dans un dilemme : la démographie galopante de la population humaine des pays en voie de développement exige une augmentation rapide et radicale de la production en protéines animales, mais le bétail autochtone n'est pas capable d'y faire face. Les produits de croisements produisent mieux que les animaux de race locale, et ils sont mieux adaptés aux mauvaises conditions climatiques et alimentaires que les animaux de race importée pure ; mais leur tolérance au parasitisme est tout au plus intermédiaire, ce qui est souvent insuffisant.

Si la tolérance envers un parasite donné est basée sur un ou sur très peu de gènes seulement, il est peut-être possible de transférer ces gènes du bétail local au bétail plus productif mais sensible. Cela pourrait se faire par des programmes de croisement et de sélection classiques ou peut-être dans un avenir plus lointain par le génie génétique. Une autre méthode possible est la sélection sur la production à l'intêrieur d'une population ou d'une race tolérante. Aucune de ces trois approches n'est rapide! Par ailleurs, toutes exigent l'existence de critères fiables de résistance et de marqueurs génétiques de tolérance, rarement disponibles à l'heure actuelle.

Le fait que le stress d'une production élevée est immunodépresseur pourrait constituer un facteur de complication, car les gènes responsables de la tolérance pourraient ne pas trouver toute leur expression chez des animaux très productifs.

\section{Lutte biologique}

Elle consiste en l'utilisation de prédateurs et de maladies de parasites. Il y a beaucoup d'exemples d'application de méthodes de lutte biologique contre des parasites de cultures. Malheureusement, cela n'est pas vrai pour les parasites d'animaux, où les exemples sont encore rares. Des possibilités théoriques nombreuses existent certes, mais la plupart n'ont pas été suivies ou bien n'ont pas abouti à des résultats pratiques. Des hyménoptères parasites ont été utilisés avec un certain succès dans la lutte contre les Stomoxys à l'île Maurice (revue par Cuisancc ct coll. (3)). Des résultats expérimentaux prometteurs ont été obtenus avec un parasitoïde contre la tique Amblyomma variegatum (9). L'utilisation de nématodes entomophages contre des tiques est à l'étude, avec des résultats prometteurs pour certains (15), bien que d'autres chercheurs soient moins enthousiastes (8). L'emploi de champignons contre les tiques Rhipicephalus appendiculatus et $A$. variegatum et contre les larves de nématodes est ćgalcment à l'étude et donne de l'espoir $(7,10)$. Néanmoins, en général les perspectives de l'application pratique de méthodes biologiques de lutte contre la plupart des parasites des animaux ne semblent pas très prometteuses dans un avenir proche.

\section{Lutte écologique}

Quelques exemples permettent d'illustrer cet aspect méthodologique :

- l'utilisation de plantes toxiques pour les tiques au pâturage ;

- la rotation des pâturages afin de priver les larves de tiques et de nématodes de leurs hôtes pendant une période suffisante pour qu'elles meurent ;

- occupation des pâturages par des animaux non sensibles sur les terrains infectés, par exemple par des chevaux ou des petits ruminants, afin de "blanchir" les tiques de la theilériose bovine, ou bien occupation des pâturages par des bovins et des petits ruminants pour diminuer la pression d'infection par Haemonchus contortus pour ces derniers ;
- les feux de brousses pour détruire des tiques, le défrichement de la végétation et la destruction du gibier pour priver les glossines du groupe morsitans d'un microclimat favorable et de leurs hôtes, donnent souvent des résultats décevants et sont actuellement devenus des méthodes pratiquement inacceptables ;

- la stabulation permanente des animaux, afin d'éviter le contact avec les tiques et les helminthes au pâturage.

Certaines des méthodes proposées sont attrayantes, mais souvent théoriques et peu pratiques. La gestion des pâturages n'est pas possible lorsqu'il s'agit de pâturages collectifs, ou de transhumance. La période pendant laquelle les larves de tiques peuvent survivre est souvent trop longue pour mériter une place dans la gestion rationnelle de l'exploitation. L'application de la rotation des pâturages serait prometteuse en climat tropical humide vis-àvis des strongyloses de petits ruminants (1).

On pourrait inclure dans lá lutte écologique l'exposition des animaux à la fumée, contre des insectes piqueurs ; elle est couramment pratiquée par des pasteurs africains.

\section{Lutte génétique}

Elle comprend des méthodes qui visent à interrompre le cycle de la reproduction des parasites par une modification de leur gènes, soit par irradiation, soit par manipulation génétique. L'approche est très spécifique et non-polluante (mise à part l'utilisation d'uné source d'irradiation). Un exemple bien connu et réussi est la technique du mâle stérile utilisée dans l'éradication de la lucilie bouchère (Cochliomyia hominivorax). Cette technique de lâcher de mâles stériles a libéré le continent nord-américain de ce fléau, et promet de libérer bientôt toute l'Amérique Centrale. Elle a récemment permis d'éliminer la lucilie bouchère de l'Afrique du Nord après son introduction accidentelle en Libye (5).

La même méthode peut être utilisée contre les glossines. A priori ces mouches s'y prêtent très bien, car elles ne sont pas nombreuses et leur pouvoir de reproduction est limité. Néanmoins, un certain nombre de facteurs a empêché de vrais súccès :

- les surfaces à traiter sont énormes et les glossines ne reconnaissent pas les frontières nationales ; une approche régionale s'impose donc ;

- le nombre important d'espèces différentes de glossines, dont l'élevage n'est techniquement acquis que pour certaines d'entre elles;

- le coût élevé de la production des mâles stériles des différentes espèces au laboratoire ;

- Ie manque d'infrastructure et de moyens financiers de la plupart des pays concernés, nécessaires pour la protection permanente des régions libérées de la mouche contre les réinvasions ; ainsi la protection des $3000 \mathrm{~km}^{2}$ libérés de trois espèces de glossines au Burkina Faso (4) n'a pas pu être maintenue ;

- aucun pays donateur n'est menacé directement par les glossines, ce qui rend difficile le financenent continu qui est absolument nécessaire. C'est une différence essentielle avec la lucilie bouchère, pour laquelle les Etats-Unis sont directement concernés. Le réchauffement inéluctable de l'atmosphère par les activités humaines pourrait changer la donne à l'avenir !

\section{Lutte mécanique}

Les pièges et les écrans imprégnés d'insecticide sont utiles contre certains ectoparasiles comme les glussines (2). Ils peuvent donner 
une partie de la solution dans certaines situations. La lutte mécanique peut être utilisée pour maintenir les vecteurs et donc la prévalence de la maladie à un bas niveau, tolérable; dans le cas des glossines, on peut mettre en place des lignes de pièges ou d'écrans servant de barrières. Les formes tridimensionnelles et l'association de couleurs attractives favorisant la pose sur le leurre (bleu et noir essentiellement) sont actuellement les plus employées. On peut les rendre encore plus attractifs par l'émission de certaines odeurs.

L'hôtc imprógné d'insecticide peut également devenir un écran vivant contre les glossines, ou un piège vivant contre les tiques en utilisant des phéromones naturelles ou synthétiques. Un leurre en plastique, incorporant des phéromones spécifiques et un acaricide, attaché à la queue des bovins, a donné des résultats prometteurs pour la lutte contre des tiques africaines du genre Amblyomma (13).

La flexibilité, l'adaptation aux conditions locales, reste primordiale. Par exemple, la lutte contre les glossines et les tiques par la méthode "pour-on" (application topique dorsale) est à considérer de façon attentive, par rapport à des résidus éventuels de l'insecticide, dans les régions humides de l'Afrique de l'Ouest où la peau constitue un mets de prédilection pour certaines populations. (B. Bauer, comm. pers., 1995).

Il est également possible de combiner la lutte génétique avec la lutte mécanique en attirant les glossines dans des pièges dont elles sont libérées après un contact avec un produit chimiostérilisant, ou un régulateur de croissance.

Un exemple bien connu de lutte mécanique en médecine humaine est l'utilisation de moustiquaires, encore plus efficaces depuis qu'elles sont imprégnées de pyréthrinoïdes.

\section{Lutte et surveillance sanitaires}

Des mesures de quarantaine peuvent prévenir la contamination, par exemple par les acariens (agents des gales) ou par les poux. Des mesures de surveillance et de quarantaine, combinées à une législation appropriée, peuvent également être utiles ou même indispensables pour empêcher la propagation ou l'importation de parasites ou de souches résistantes aux pesticides.

Il existe actuellement un programme pour l'éradication de la tique Amblyomma variegatum des Antilles, tique africaine introduite involontairement dans les îles de la mer des Caraïbes, d'où elle menace le continent américain. Des mesures de surveillance s'appuyant sur une législation pertinente doivent faire partie inté grante de ce programme.

\section{- CONCLUSION}

Plusieurs raisons irréfutables plaident en faveur d'une lutte intégrée, flexible et adaptée aux circonstances locales, contre les parasitoses des animaux domestiques. Bien que son application reste relativement limitée pour l'instant, il y a de nombreuses possibilités à explorer. La durabilité de la lutte intégrée est essentielle, aussi bien du point de vue économique que de celui de la protection de l'environnement.

\section{REFERENCES}

1. BARGER I.A., SIALE K., BANKS D.J.D., LE JAMBRE L.F., 1994. Rotational grazing for control of gastrointestinal nematodes of goats in a wet tropical environment. Vet. Parasitol., 53: 109-116.
2. CUISANCE D., 1989. Le piégeage des tsé-tsé. Maisons-Alfort, France, CIRAD-IEMVT, 172 p. (Etudes et Synthèses de I'IEMVT n ${ }^{\circ}$ 32)

3. CUISANCE D., BARRE N., DE.DEKEN R., 1994. Ectoparasites des animaux : méthodes de lutte écologique, biologique, génétique et mécanique. Revue sci. tech. Off. int. Epiz., 13 : 1305-1356.

4. CUISANCE D., POLITZAR II., MLROT P., TAMBOURA I., 1984. Les lâchers de mâles irradiés dans la campagne de lutte intégrée contre les glossines dans la zone pastorale de Sidéradougou (Burkina Faso). Revue Elev. Méd. vét. Pays trop., 37 : 449-467.

5. FAO, 1992. The new world screwworm eradication programme. North Africa 1988-1992. Rome, Italy, FAO, 192 p.

6. FRISCH J.E., 1994. Identification of a major gene for resistance to cattle ticks. Proc. 5th World Congr. Genet. appl. Livest. Prod., 20: 293295.

7. KAAYA G.P., MWANGI E.N., OUNA, E. Prospects of biological control of livestock licks, Rhipicephalus appendiculatus and Amblyomma variegatum with the entomogenous fungi Beauveria bassiana and Metarhizium anisopliae. J. Invertebr. Pathol. (In press)

8. MAULEON H., BARRE N., PANOMA S., 1993. Pathogenicity of 17 isolates of entomophagous ncmatodes (Steinernematidae and Heterorhabditidae) for the ticks Amblyomma variegatum (Fabricius), Boophilus microplus (Canestrini) and Boophilus annulatus (Say). Exp. appl. Acarol., 17: 831-838.

9. MWANGI F.N., KAAYA G.P., ESSUMAN S., 1994. Parasitism of Amblyomma variegatum by a hymenopteran parasitoid and some aspects of its basic biology. J. Biol. Control, 4: 101-104.

10. NANSEN P., GRONVOLD J., WOISTRUP M., LARSEN M., HENRIKSEN S.A., 1994. Attempts to control nematode parasites in cattle by nematode-trapping fungi - a survey. In: Animal diseases of the gastrointestinal tract and liver: an African perspective. Stockholm, Sweden, International Foundation for Science, p. 35-42.

11. NJOGU A.R., DOLAN R.B., WILSON A.J., SAYER P.D., 1985. Trypanotolerance in East African Orma Boran cattle. Vet. Rec., 117: 632-636.

12. NORVAL R.A.I., 1979. Tick infestations and tick-borne diseases in Zimbabwe. J. S. Afr. vet. Assoc., 50: 289-292.

13. NORVAL R.A.I., SONENSHINE D.E., ALLAN S.A., BURRIDGE M.J., 1996. Efficacy of pheromone-acaricide-impregnated tail-tag decoys for controlling the bont tick, Amblyomma hebraeum (Acari: Ixodidae), on cattle in Zimbabwe. Exp. appl. Acarol., 20: 31-46.

14. OIE., 1994. Ectoparasites of animals and control methods, Revue sci. tech. Off. int. Epiz., 13: 979-1365.

15. SAMISH M., GLAZER I., 1992. Infectivity of entomopathogenic nematodes (Steinernematidae and Heterorhabditidae) to female ticks of Boophilus annulatus (Arachnida: Ixodidae). J. med. Entomol., 29: 614618.

16. UILENBERG G., 1995. Lutte intégrée contre les parasitoses animales tropicales. In : Congrès d'hiver 1995-1996, Société française de parasitologie, Ecole nationale vétérinaire d'Alfort, France, 7-8 décembre 1995. Maisons-Alfort, France, Ecole nationale vétérinaire, p. 171-172. (Résumés des confërences et communications)

17. YOUNG A.S., GROOCOCK C.M., KARIUKI D.P., 1988. Integrated control of ticks and tick-borne diseases of cattle in Africa. Parasitology, 96: 403-432.

Reçu le 13.2.96, aucepté le 30.4.96 


\section{Summary}

Uilenberg G. Integrated control of tropical animal parasitoses

In the past, parasite control in domestic animals has relicd mainly on the use of drugs and pesticides. Although these compounds are still of great importance in the prevention and treatment of parasitic diseases, in recent years the emphasis has shifted to a more flexible approach, integrating various other control measures. The main reasons for this change are:

- development of parasite resistance to the compounds used;

- reduced development of new compounds to overcome resistance (increasingly more stringent regulations on toxicity and residues, resulting in very high research and develupment costs, insufficient return for industry because of the short lifespan of new products due to resistance and berause the market for compounds in developing countries is limited and poor);

- increasing cost of new products for consumers;

- problems associated with toxicity, environmental pollution and residues in animal products.

Integrated parasite management makes use, where possible, of biological and mechanical control, of acquired and innate host resistance, and genetical, ecological, sanitary and regulatory procedures, although chemical control can seldom be entirely eliminated. Cost-effectiveness and sustainability in all respects are of primary importance.

Key words: Animal parasitosis - Integrated control - Chemical resistance - Cost - Environment - Profitability - Developing country.

\section{Resumen}

Uilenberg G. Lucha integrada contra las parasitosis animales tropicales

En el pasado, la lucha contra los parásitos se basaba esencialmente en el uso de medicamentos y pesticidas. Aunque estos compuestos tienen aún una gran importancia en la prevención y el tratamiento de las enfermedades parasitarias, la tendencia actual se orienta hacia un enfoque más flexible, intengrando diversos medios de combate contra las patologías. Las razones principales de esta evolución son las siguientes :

- aparición de resistencias parasitarias a los compuestos utilizados ;

- retraso en el desarrollo de los nuevos compuestos para compensar este fenómeno de resistencia (reglamentación más estricta sobre la toxicidad y los residuos, con costos de investigación y de desarrollo muy elevados, con una rentabilidad insuficiente para la industria, debido al período de vida limitado de los nuevos productos, causado a su vez por los fenómenos de resistencia y por el pobre y limitado mercado de los compuestos en los países en vía de desarrollo);

- aumento del costo de los nuevos productos para el utilizador ;

- problemas ligados a la toxicidad, a la polución del medio ambiente y a los residuos en los productos de origen animal.

El manejo integrado de los medios de lucha contra los parásitos utiliza, en la medida de lo posible, la lucha biológica y mecánica, la resistencia adquirida y propia del huésped del parásito, así como procedimientos mecánicos, sanitarios y de reglamentación, mismo si la lucha química es rara vez eliminada por completo. Es primordial prestar una atención particular a la rentabilidad y al carácter durable, en todos los sentidos, de los medios de lucha utilizados.

Palabras clave : Parasitosis de animales - Lucha integrada Resistencia química - Costo - Medio ambiente - Rentabilidad - País en desarrollo. 